\title{
A Model-Informed Method for the Purpose of Precision Dosing of Isoniazid in Pulmonary Tuberculosis
}

\author{
Stijn W. van Beek ${ }^{1} \oplus \cdot$ Rob ter Heine $^{1} \cdot$ Jan-Willem C. Alffenaar ${ }^{2,3,4} \cdot$ Cecile Magis-Escurra $^{5} \cdot$ Rob E. Aarnoutse $^{1}$. \\ Elin M. Svensson ${ }^{1,6}$ on behalf of the Isoniazid Precision Dosing Group
}

Accepted: 28 November 2020 / Published online: 22 February 2021

(c) The Author(s) 2021

\begin{abstract}
Background and Objective This study aimed to develop and evaluate a population pharmacokinetic model and limited sampling strategy for isoniazid to be used in model-based therapeutic drug monitoring.

Methods A population pharmacokinetic model was developed based on isoniazid and acetyl-isoniazid pharmacokinetic data from seven studies with in total 466 patients from three continents. Three limited sampling strategies were tested based on the available sampling times in the dataset and practical considerations. The tested limited sampling strategies sampled at 2, 4, and $6 \mathrm{~h}, 2$ and $4 \mathrm{~h}$, and $2 \mathrm{~h}$ after dosing. The model-predicted area under the concentration-time curve from 0 to $24 \mathrm{~h}$ $\left(\mathrm{AUC}_{24}\right)$ and the peak concentration from the limited sampling strategies were compared to predictions using the full pharmacokinetic curve. Bias and precision were assessed using the mean error (ME) and the root mean square error (RMSE), both expressed as a percentage of the mean model-predicted $\mathrm{AUC}_{24}$ or peak concentration on the full pharmacokinetic curve. Results Performance of the developed model was acceptable and the uncertainty in parameter estimations was generally low (the highest relative standard error was 39\% coefficient of variation). The limited sampling strategy with sampling at 2 and $4 \mathrm{~h}$ was determined as most suitable with an ME of $1.1 \%$ and RMSE of $23.4 \%$ for $\mathrm{AUC}_{24}$ prediction, and ME of $2.7 \%$ and RMSE of $23.8 \%$ for peak concentration prediction. For the performance of this strategy, it is important that data on both isoniazid and acetyl-isoniazid are used. If only data on isoniazid are available, a limited sampling strategy using 2,4 , and $6 \mathrm{~h}$ can be employed with an ME of $1.7 \%$ and RMSE of $20.9 \%$ for $\mathrm{AUC}_{24}$ prediction, and $\mathrm{ME}$ of $1.2 \%$ and RMSE of $23.8 \%$ for peak concentration prediction.

Conclusions A model-based therapeutic drug monitoring strategy for personalized dosing of isoniazid using sampling at 2 and $4 \mathrm{~h}$ after dosing was successfully developed. Prospective evaluation of this strategy will show how it performs in a clinical therapeutic drug monitoring setting.
\end{abstract}

Stijn W. van Beek

Stijn.vanBeek@radboudumc.nl

1 Department of Pharmacy, Radboud Institute for Health Sciences, Radboud University Medical Center, Geert Grooteplein zuid 10, 864, $6500 \mathrm{HB}$, Nijmegen, The Netherlands

2 School of Pharmacy, Faculty of Medicine and Health, University of Sydney, Sydney, NSW, Australia

3 Westmead Hospital, Sydney, NSW, Australia

4 Marie Bashir Institute of Infectious Diseases and Biosecurity, University of Sydney, Sydney, NSW, Australia

5 Department of Respiratory Diseases, Radboud University Medical Center, Nijmegen, The Netherlands

6 Department of Pharmacy, Uppsala University, Uppsala, Sweden

\section{Key Points}

Model-informed precision dosing may aid in optimizing tuberculosis treatment

We developed a population pharmacokinetic model for isoniazid and its metabolite based on the largest and most diverse dataset thus far and thoroughly validated its suitability for purposes of dose individualization

We found that sampling at 2 and $4 \mathrm{~h}$ with data on both isoniazid and acetyl-isoniazid, or sampling at 2, 4, and $6 \mathrm{~h}$ without the metabolite could be used to predict exposure (area under the concentration-time curve) 


\section{Background}

Although the disease burden of tuberculosis (TB) is falling, it remains one of the top ten causes of death globally and is the leading cause of death from a single infectious agent [1]. The average global treatment success rate for drugsusceptible TB was estimated at 85\% in 2017 [1]. Despite this high success rate, it still means that one out of every seven patients treated for TB has an unfavorable treatment outcome. This shows that a significant part of the patients does not respond adequately to treatment. A possible reason for not responding to treatment is suboptimal anti-TB drug exposure [2].

Isoniazid (INH) is one of the pillars on which treatment of drug-susceptible TB is based and is listed as an essential medicine by the World Health Organization [3]. Low INH concentrations compared to the median in a population have been reported to be common and are often a result of high inter-individual variability (IIV) in the pharmacokinetics of this drug [4-7]. A large part of this variability can be attributed to the polymorphic $\mathrm{N}$-acetyltransferase 2 (NAT2) enzyme that catalyzes the acetylation of INH into its acetylINH metabolite [8].

Large inter-individual variability in pharmacokinetics is one of the motives and prerequisites for therapeutic drug monitoring (TDM) [2]. Therapeutic drug monitoring is an approach to personalize drug dosing by measuring a patient's drug exposure and comparing it to a drug-specific target level and adjusting the dose if needed. The exposure is approximated by sampling at drug-specific planned times. This strategy is called a limited sampling strategy (LSS). The area under the concentration-time curve over a dosing interval (0-24 h, $\left.\mathrm{AUC}_{24}\right)$ in relation to a minimal inhibitory concentration has been proposed to be the most important predictor of treatment outcome for the first-line TB drugs [9-12]. However, many different pharmacokinetic (PK) target suggestions exist [2, 9, 13-16]. Some of these have a peak concentration $\left(C_{\max }\right)$ target while others use $\mathrm{AUC}_{24}$. Lacking anything more precise, a population median AUC 24 value of INH corrected for acetylator status could serve as a PK target as it is known that the standard dose is generally effective [2].

We propose the use of a model-based TDM method using individual predictions from a population PK model for INH. Using a model-based method for TDM has several advantages: it can use flexible sampling times, it allows exposure predictions following dose adjustments, and it can use data from previous sampling occasions for the same patient in the prediction. Available models for INH are often based on data from a single-center study with a limited sample size limiting the generalizability of their use for dose individualization $[4,5,17-22]$. The aim of this study was to develop and evaluate a model-based TDM approach for INH in adult patients with pulmonary TB using a population-PK model suitable for dose individualization in programmatic treatment.

\section{Methods}

\subsection{Data}

The data originated from seven previously conducted studies, including data from both clinical trials and TDM [14, 23-28]. All studies included patients with pulmonary TB. The study data are presented in Table 1. In total, data of 466 patients from three continents were used to develop the PK model. These PK data consisted of 2546 INH and 2236 acetyl-INH concentrations collected during an interval range of $0-24 \mathrm{~h}$. Of these concentrations, $6.0 \%$ of the INH and $10.9 \%$ of the acetyl-INH observations were measured as 'below the limit of quantification' (BLQ), which ranged from 0 to $27.3 \%$ depending on the study.

Four studies involved intensive PK sampling with at least nine samples taken after a dose of INH [14, 24, 26, 27], two studies involved limited sampling with two to three samples taken [23, 28], and one study involved limited sampling with one sample taken for all except nine individuals who were sampled intensively [25]. Five studies only included measurements of a single occasion [14, 24-27], one study included some measurements of second occasions [28], and one included measurements for second occasions for all individuals [23]. One study included data that were collected during TDM practice [28]. Five studies included PK data on both INH and acetyl-INH [24-28], while the other two studies included PK data on INH [14, 23].

The analysis of INH and acetyl-INH concentrations was performed in The Netherlands for all studies, using either ultra-performance liquid chromatography with ultraviolet detection or liquid chromatography-tandem mass spectrometry [29]. Both methods were extensively validated in accordance with European Medicines Agency and US Food and Drug Administration guidelines. Accuracy was between 99.4 and $108.8 \%$ and between 95.6 and $111.3 \%$ and the intra- and inter-assay coefficients of variation $(\mathrm{CVs})$ were less than $12.6 \%$ and $10.5 \%$ for the ultra-performance liquid chromatography with ultraviolet detection and liquid chromatography-tandem mass spectrometry methods, respectively, dependent on the concentration. The lower limits of quantification for INH and acetyl-INH for the ultra-performance liquid chromatography method were $0.0253 \mathrm{mg} / \mathrm{L}$ and $0.162 \mathrm{mg} / \mathrm{L}$, and these were 0.0452 $\mathrm{mg} / \mathrm{L}$ and $0.0450 \mathrm{mg} / \mathrm{L}$ for the liquid chromatographytandem mass spectrometry method. Performance of the 
Table 1 Overview of the included study data

Semvua [23] Tostmann [24] Burhan [25] Aarnoutse [26] $\quad$ Magis-Escurra Boeree [27] Van Beek [28] Total [14]

\begin{tabular}{|c|c|c|c|c|c|c|c|c|}
\hline $\begin{array}{l}\text { Included } \\
\text { patients }\end{array}$ & 23 & 40 & 180 & 62 & 14 & 97 & 52 & 466 \\
\hline \multicolumn{9}{|c|}{ Observed data points } \\
\hline Total & 92 & 619 & 534 & 1189 & 154 & 1804 & 390 & 4782 \\
\hline INH & 92 & 309 & 263 & 619 & 154 & 898 & 211 & 2546 \\
\hline Acetyl-INH & 0 & 310 & 271 & 570 & 0 & 906 & 179 & 2236 \\
\hline \multicolumn{9}{|c|}{ Data points BLQ, $n(\%)$} \\
\hline Total & $2(2.2)$ & $54(8.7)$ & $1(0.2)$ & $40(3.4)$ & $42(27.3)$ & $249(13.8)$ & $7(1.8)$ & $395(8.3)$ \\
\hline INH & $2(2.2)$ & $27(8.7)$ & $0(0)$ & $5(0.8)$ & $42(27.3)$ & $72(8.0)$ & $4(1.9)$ & $152(6.0)$ \\
\hline Acetyl-INH & - & $27(8.7)$ & $1(0.4)$ & $35(6.1)$ & - & $177(19.5)$ & $3(1.7)$ & $243(10.9)$ \\
\hline $\begin{array}{l}\text { Typical sam- } \\
\text { pling hours }\end{array}$ & 2 and $6 \mathrm{~h}$ & $\begin{array}{l}\text { Full PK, } 9 \\
\text { samples } \\
0-24 \mathrm{~h}\end{array}$ & $\begin{array}{l}2 \mathrm{~h} \text {, full PK } \\
\text { for } 9 \text { patients }\end{array}$ & $\begin{array}{l}\text { Full PK, } 11 \\
\text { samples } \\
0-24 \text { h }\end{array}$ & $\begin{array}{c}\text { Full PK, } 11 \\
\text { samples } \\
0-24 \mathrm{~h}\end{array}$ & $\begin{array}{c}\text { Full PK, } 9 \\
\text { samples } \\
0-24 \mathrm{~h}\end{array}$ & 2,4 , and $6 \mathrm{~h}$ & - \\
\hline $\begin{array}{c}\text { Samples, } \\
\text { median } \\
\text { (range) }\end{array}$ & $4(4-4)$ & $8(4-9)$ & $1(1-13)$ & $10(7-11)$ & $11(10-11)$ & $9(5-10)$ & $4(1-8)$ & - \\
\hline $\begin{array}{l}\text { Number of } \\
\text { occasions }\end{array}$ & 2 & 1 & 1 & 1 & 1 & 1 & $\begin{array}{r}1 \text { ( } 2 \text { for } 14 \\
\text { patients) }\end{array}$ & - \\
\hline $\begin{array}{l}\text { Bioanalysis } \\
\text { method }\end{array}$ & UPLC-UV & UPLC-UV & UPLC-UV & UPLC-UV & LC-MS/MS & UPLC-UV & LC-MS/MS & - \\
\hline $\begin{array}{l}\text { Country of } \\
\text { origin }\end{array}$ & Tanzania & Tanzania & Indonesia & Tanzania & $\begin{array}{l}\text { The } \\
\text { Netherlands }\end{array}$ & $\begin{array}{l}\text { Tanzania and } \\
\text { South Africa }\end{array}$ & $\begin{array}{l}\text { The } \\
\text { Netherlands }\end{array}$ & - \\
\hline Setting & Clinical trial & Clinical trial & Clinical trial & Clinical trial & TDM & Clinical trial & TDM & - \\
\hline
\end{tabular}

$B L Q$ below limit of quantification, $h$ hour, $I N H$ isoniazid, $L C$ - $M S$ or liquid chromatography-mass spectrometry, $P K$ pharmacokinetics, $T D M$ therapeutic drug monitoring, UPLC ultra-performance liquid chromatography

${ }^{a}$ While the patients from these studies were treated in The Netherlands, the TB population in The Netherlands is very heterogenous with many different ethnical backgrounds

assays was externally evaluated by participation in an international proficiency testing program [30].

\subsection{Software}

$\mathrm{R}$ version 3.4.3 was used for data management, statistics, and plotting [31]. Model development was performed using the nonlinear mixed-effects modeling program, NONMEM version 7.4 with Pirana as an interface $[32,33]$. The firstorder conditional estimation method with interaction was used for estimation. PsN version 4.7 was used as an aid for advanced functionalities [33]. The Xpose4 R package version 4.6.1 was used for graphical visualization of the visual predictive checks (VPCs) [33].

\subsection{Pharmacokinetic Model Development}

The PK model was developed with a stepwise approach [34]. The INH concentration data were included one study at a time, starting with the studies that had the most informative and dense sampling. After finishing the base INH model, the acetyl-INH data were added to the model. One- and twocompartment disposition models were explored to describe the pharmacokinetics of INH and acetyl-INH. A well-stirred liver model was tested instead of first-order elimination to describe the first-pass effect of INH. The molecular weight of INH and acetyl-INH was used to transform drug concentrations to molar concentrations to model the formation of acetyl-INH from INH. The bioavailability of INH was assumed to be $100 \%$ and all estimated PK parameters should be interpreted as apparent oral PK parameters. All volume and clearance parameters were allometrically scaled with total body weight using an exponent of 1 or 0.75 , respectively [35]. Allometric scaling based on fat-free mass was not considered because height is not always readily available in TDM practice. NAT2 genotypic data were not determined in the included studies. A mixture model to distinguish between different groups of NAT2 metabolizers in the absence of genotypic data was applied [36]. It is known that the distribution of NAT2 metabolic function is trimodal [8]. The mixture model was tested with two as well as three 
metabolizer groups as it can be difficult to distinguish the three mixture groups as is seen in other models $[4,17]$. In case two, metabolizer groups were used, the fast and intermediate metabolizers were grouped, as metabolization rates of these groups are closer together than those of the slow metabolizers, which have more deviant metabolization rates.

Inter-individual variability in the pharmacokinetics of INH was assumed to be log-normally distributed. Interoccasion variability was not assessed because the number of individuals who were sampled on multiple occasions was limited. For residual variability, additive, proportional, and combined models were tested for both INH and acetyl-INH. Below the limit of quantification data were handled using the M6 method as described by Beal [37]. The M3 method was tested but did not perform better than the M6 method and increased numerical instability. Using this method, in the elimination phase, the first datapoint BLQ is replaced by the lower limit of quantification/2, and subsequent BLQ points are ignored. In the absorption phase, it is the last datapoint BLQ that is replaced and all previous BLQ points that are ignored. The minimum additive error for data BLQ was fixed to $50 \%$ of the lower limit of quantification values.

The number of potential covariates tested was kept low because of the purpose of our model; the covariates available in TDM practice are generally quite limited. It was opted to include only the allometric scaling with total body weight to not hamper a general implementation of the model in routine TDM. Although we did test the impact of formulation differences, but no effect could be identified.

Pharmacokinetic parameter uncertainty was assessed using sampling importance resampling [38, 39]. Sampling importance resampling is a fast method to determine parameter uncertainty which is free of distributional assumptions, a good alternative to the bootstrap. The initial estimates for the SIR were based on a successful covariance step and inflated by factor 1.5 . The samples per iteration were 1000 , $1000,1000,2000$, and 2000, the re-samples per iteration were $200,400,500,1000$, and 1000. Parameter precision was presented using the calculated $95 \%$ confidence intervals (CIs). The predictive performance of the final model was assessed using VPCs and goodness-of-fit plots. The VPCs were prediction corrected and the PsN mixture option was used $[40,41]$.

\subsection{Comparison of Limited Sampling Strategies}

The performance of three LSSs was tested. These LSSs had sampling times at $2 \mathrm{~h}, 2$ and $4 \mathrm{~h}$, and 2, 4, and $6 \mathrm{~h}$ after dosing. These LSSs were chosen considering that sampling at 2 and $6 \mathrm{~h}$, introduced by Peloquin et al., is common for TB drugs, and we have added a 4-h sampling point in our TDM service [2, 42]. In addition, samples with these sampling times were available within most of the datasets. Sampling at 2 and $6 \mathrm{~h}$ was not included as it does not offer any benefit over sampling at 2 and $4 \mathrm{~h}$, which is more feasible to perform in clinical practice. For each LSS, the performance of $\mathrm{AUC}_{24}$ and $C_{\max }$ prediction was determined using the patients who had a full PK curve including samples at 2, 4, and $6 \mathrm{~h}$ after dosing (169 patients from five studies) [14, 24-27]. The performance of the LSSs was also compared to the performance of the model without any sampling to assess the added value of sampling in TDM. The most suitable LSS was selected based on predictive performance and clinical practicality. The performance of predicting the $\mathrm{AUC}_{24}$ was regarded as more important than that of the $C_{\max }$. In these assessments, the mean error (ME) and root mean square error (RMSE) and their 95\% CIs of the prediction of $\mathrm{AUC}_{24}$ and $C_{\max }$ were calculated as measures of bias and precision $[43,44]$. The ME and RMSE were expressed as a percentage of the mean of the corresponding model-estimated parameter using the full PK curve.

The most suitable LSS was used to perform a fit-for-purpose analysis, meaning that it was investigated how well the pharmacokinetics of INH could be predicted from a previous measurement [45]. The patients including data of a second occasion and the sampling times for the selected LSS were used for this. Apart from the most suitable LSS, 'no sampling' was tested as well to evaluate the amount of variability explained by the most suitable LSS. Suitable data for this analysis were available from 13 patients from only one study [28]. The performance of predicting the $\mathrm{AUC}_{24}$ for the second occasion was determined using the ME and RMSE.

\subsection{Importance of Acetyl-INH Data and Mixture Component for the Predictive Performance}

The added value of both the acetyl-INH metabolite data and inclusion of a mixture model accounting for the polymorphic NAT2 clearance was assessed for the most suitable LSS. The selected LSS was used to provide $\mathrm{AUC}_{24}$ predictions using only the INH data. These predictions were compared to model predictions using the full dataset, including the acetyl-INH data. To determine the added benefit of a mixture model to the performance of predicting the $\mathrm{AUC}_{24}$, the mixture component was removed, and model parameters were re-estimated. The performance was again compared using the ME and RMSE.

\section{Results}

\subsection{Pharmacokinetic Model}

The final model is depicted in Fig. 1. It included four transit absorption compartments going into a well-stirred liver model [46]. The pharmacokinetics of INH was best described by 
a two-compartment disposition model. Acetyl-INH pharmacokinetics was characterized using a single compartment and first-order elimination. Metabolization in the liver compartment could either clear INH or transform it into acetyl-INH. It was not possible to estimate INH clearance as a trimodal distribution using a mixture model and thus a bimodal mixture model was included. The volume of the liver compartment and hepatic plasma flow were fixed to $1 \mathrm{~L}$ and $49.5 \mathrm{~L} / \mathrm{h}$, respectively, and allometrically scaled on total body weight like other volume and clearance parameters.

The final model parameter estimates and their uncertainty are shown in Table 2. Uncertainty in the parameter estimates was generally low (relative standard error, RSE $<12 \% \mathrm{CV}$ ), except for the IIV of the central volume of acetyl-INH (RSE = $40 \% \mathrm{CV}$ ) and the correlation between the IIV of the clearances $(\mathrm{RSE}=71 \% \mathrm{CV})$. The absorption processes were variable between individuals with an IIV estimated at $83.2 \% \mathrm{CV}(95 \%$ CI 78.1-91.7). The proportion of fast NAT2 acetylators in the studies was estimated at 43.4\% (95\% CI 38.4-48.9). Slow acetylators typically had $13 \%$ of the intrinsic NAT2 clearance of fast acetylators. The second clearance pathway, not through NAT2, typically made up $74 \%$ of the total clearance for slow acetylators and $27 \%$ of the clearance for fast acetylators. A correlation was found between the clearance through acetylation and through other pathways, but the uncertainty of this correlation was quite high $(0.143,95 \%$ CI $-0.0620,0.325)$. Additionally, a correlation between the proportional residual errors of INH and acetyl-INH was found.

The VPCs, shown in Fig. 2, generally describe the model well. Some limitations of the model can be discerned from this figure. The model does not describe the median observed peak perfectly. Furthermore, the model simulates concentrations that are slightly too high on the upper end of the concentration range for acetyl-INH at timepoints after $8 \mathrm{~h}$. The VPCs of the model were deemed satisfactory for TDM using the $\mathrm{AUC}_{24}$. The performance of the model for TDM using the $C_{\max }$ should be explored further. Other goodness-of-fit plots are shown in Fig. 3 and do not show any obvious misspecifications. The full code of the final model is included in the Electronic Supplementary Material (ESM).

\subsection{Comparison of Limited Sampling Strategies}

The $\mathrm{AUC}_{24}$ and $C_{\max }$ predicted by the LSSs were compared to the $\mathrm{AUC}_{24}$ and $C_{\max }$ predicted by the model based on full PK curves. Scatterplots of this are shown in ESM 1. The bias and precision of these strategies are shown in Table 3. The LSS using only one sample performed substantially worse in both bias and precision than the strategies with two or three sampling points. The strategies employing sampling at 2 and 4, and 2, 4, and 6 hours after dosing have a similar performance. Based on bias, precision, clinical practicality, and conformity with a previously developed strategy for rifampicin [28], the strategy using sampling at 2 and 4 hours after dosing was selected as the most suitable.

To assess if the model and LSS is fit for purpose, the $\mathrm{AUC}_{24}$ of a second sampling occasion was predicted using data from a first sampling occasion. The fit-for-purpose analysis of the model using the 2- and 4-h sampling strategy resulted in an ME of $25.7 \%$ and an RMSE of $37.2 \%$. Without

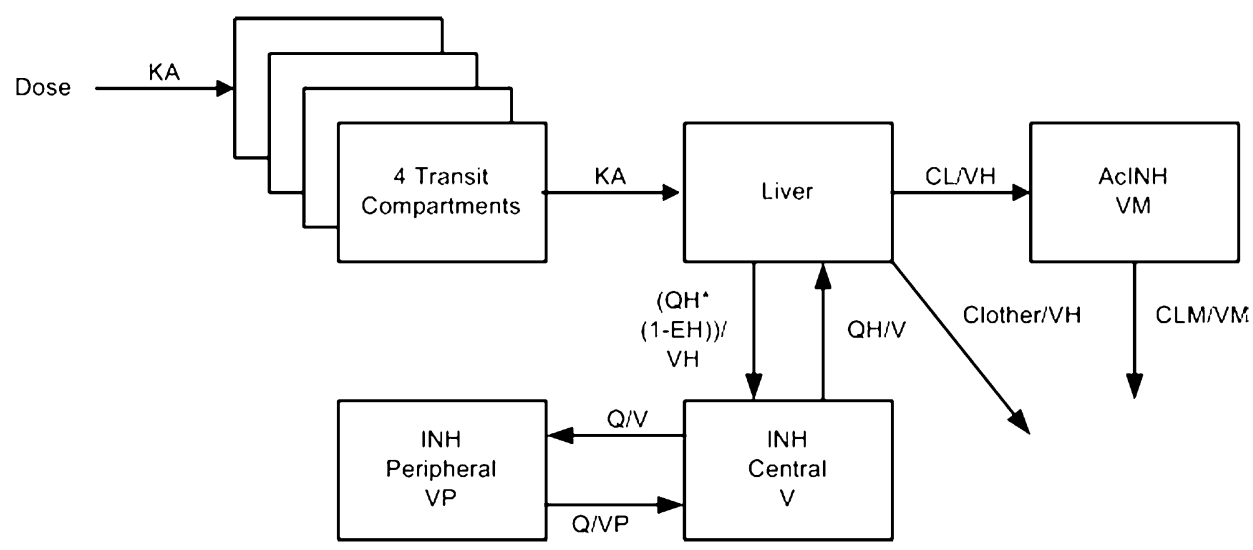

Fig. 1 Schematic overview of the final isoniazid (INH) population pharmacokinetic model. The dose enters the well-stirred liver compartment via a four-compartmental transit model. Absorption constant (KA) is defined as the number of transit compartments plus 1 , divided by the mean transit time (MTT). From the liver compartment, the drug can be distributed to the central INH compartment or be metabolized into acetyl-isoniazid (AcINH) or other metabolites. From the central AcINH compartment, the drug is cleared through firstorder elimination. Isoniazid pharmacokinetics are described using a two-compartment disposition model and AcINH pharmacokinetic by a one-compartment disposition model. $C L$ clearance into acetylisoniazid, $C L$ other clearance into other metabolites than acetyl-isoniazid, $C L M$ clearance of the acetyl-isoniazid metabolite, $E H$ hepatic extraction ratio, $Q$ inter-compartmental clearance, $Q H$ hepatic plasma flow, $V$ central volume of isoniazid, $V H$ hepatic volume, $V M$ central volume of the acetyl-isoniazid metabolite, $V P$ peripheral volume of isoniazid 
Table 2 Final model parameter estimates

\begin{tabular}{lcll}
\hline Parameter & Estimate & $95 \%$ CI & RSE (\%CV) \\
\hline Isoniazid & & & 2.2 \\
Central volume, V (L) & 57.5 & $54.9-59.4$ & 0.1 \\
Absorption constant, KA (/h) & 5.42 & $5.40-5.44$ & 6.6 \\
Proportion fast acetylators & 0.434 & $0.384-0.489$ & \\
Clearance (L/h) & & & 7.7 \\
Fast acetylators, CLF & 32.7 & $28.6-37.6$ & 7.4 \\
Slow acetylators, CLS & 4.31 & $3.85-4.75$ & 4.3 \\
Other clearance pathways, CLO & 12.1 & $11.2-13.1$ & 4.2 \\
Peripheral volume, VP (L) & 18.7 & $16.8-20.5$ & 8.2 \\
Inter-compartmental clearance, Q (L/h) & 2.48 & $2.10-2.87$ & 6.5 \\
IIV V (\%CV) & 26.4 & $23.4-29.3$ & 5.9 \\
IIV KA (\%CV) & 83.2 & $78.1-91.7$ & 5.1 \\
IIV CLF/CLS (\%CV) & 57.5 & $51.9-63.3$ & 3.0 \\
IIV CLO (\%CV) & 37.9 & $34.4-41.0$ & \\
Proportional error (\%) & 37.5 & $36.4-38.7$ & 4.3 \\
Acetyl-isoniazid & & & 4.5 \\
Central volume, VM (L) & 39.2 & $36.1-42.2$ & 37 \\
Clearance, CLM (L/h) & 6.65 & $6.10-7.22$ & 5.3 \\
IIV VM (\%CV) & 10.3 & $5.98-14.2$ & 2.8 \\
IIV CLM (\%CV) & 36.7 & $34.2-40.4$ & \\
Proportional error (\%) & 23.3 & $22.8-24.0$ & \\
Correlations & & $-0.0620 ; 0.325$ & $0.556-0.656$ \\
Acetylation-other clearance pathways & 0.143 & & \\
Parent error-metabolite error & 0.601 & & \\
\hline & & & \\
\end{tabular}

$C I$ confidence interval, $C V$ coefficient of variation, $h$ hour, $I I V$ inter-individual variability, $L$ liter, RSE relative standard error

${ }^{a}$ The clearances for fast and slow acetylators shared one estimated IIV sampling in the first occasion, the ME was $82.7 \%$ and the RMSE $117.9 \%$.

\subsection{Importance of Acetyl-INH Data and Mixture Component for the Predictive Performance}

The final model using the 2- and 4-h LSS without the acetylINH data had an ME of $-1.8 \%$ and an RMSE of $28.9 \%$ for estimating the $\mathrm{AUC}_{24}$. For estimation of the $C_{\max }$, the ME was $0.1 \%$ and the RMSE $23.5 \%$. Comparison to data in Table 3 shows that the prediction of the model without acetyl-INH data is less precise compared to the predictions including acetyl-INH for the 2- and 4-h sampling strategy. For the 2-, 4-, and 6-h strategy without acetyl-INH data, the estimation of the $\mathrm{AUC}_{24}$ had an ME of $1.7 \%$ and an RMSE of $20.9 \%$. For estimation of the $C_{\max }$, the ME was $1.2 \%$ and the RMSE $23.8 \%$. This is comparable to the performances of the 2-, 4-, and 6-h and 2- and 4-h strategies including acetyl-INH data. ESM 2 a shows the effect of removing the acetyl-INH data on the performance of predicting the AUC ${ }_{24}$ for the 2- and 4-h sampling strategy.
The re-estimated model without a mixture component using the 2- and 4-hour LSS had an ME of -1.1\% and RMSE of $24.6 \%$ for $\mathrm{AUC}_{24}$ estimation, and an ME of 7.7\% and RMSE of $23.3 \%$ for estimation of the $C_{\max }$, meaning that the mixture component was not essential for the predictive performance of the model. The estimated PK parameters for the model without a mixture component were similar to those of the final model, except for the clearance representing both metabolization groups and their IIV. The new clearance has a value of $9.12 \mathrm{~L} / \mathrm{h}$ and lies in between the clearances of the slow and fast metabolizers as estimated by the final model. The estimated IIV of this clearance has increased drastically by more than two-fold to $115.8 \% \mathrm{CV}$. ESM $2 b$ shows the effect of removing the mixture model on the performance of predicting the $\mathrm{AUC}_{24}$.

\section{Discussion}

In this project, we have developed a model describing the pharmacokinetics of INH based on a large and diverse dataset. The model should have a wider applicability and be 


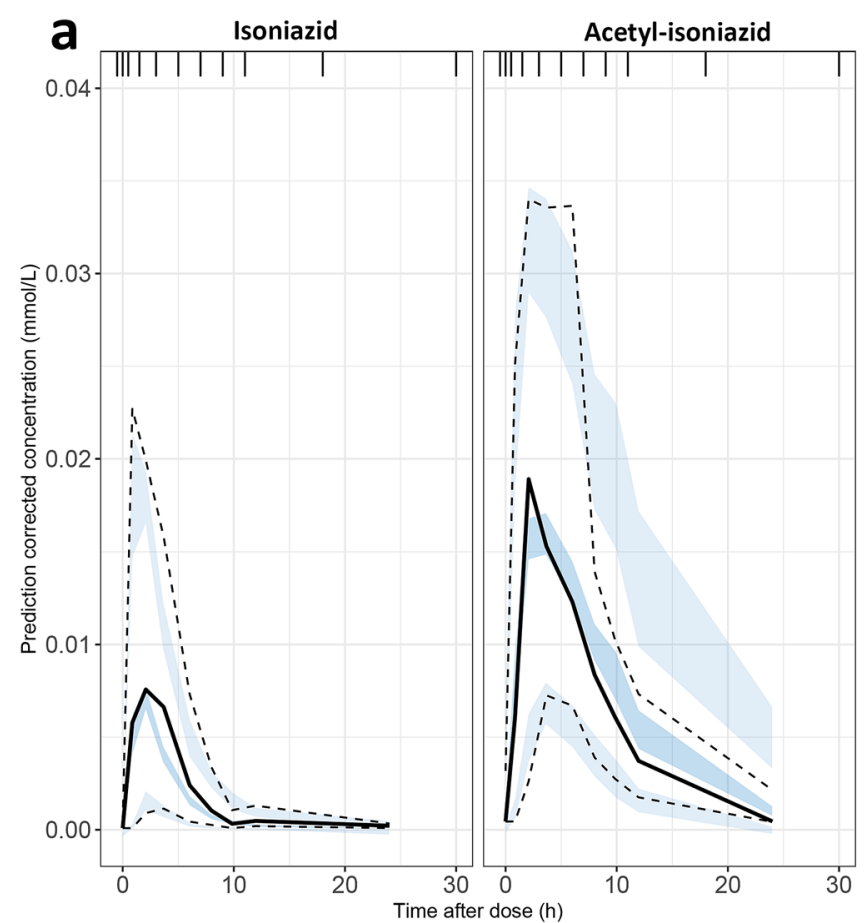

Fig. 2 Basic goodness-of-fit plots for the final isoniazid population pharmacokinetic model. a, b The observed concentrations plotted against the model-predicted concentrations or the individual modelpredicted concentrations. The black lines represent the line of unity, and the blue lines represent the local polynomial regression fit. $\mathbf{c}$ The conditionally weighted residuals plotted against the time after dose.

better suited for TDM in various populations compared to previously published models, which were all based on studies from a single country $[4,5,17-22]$. We also introduced and evaluated a model-based TDM approach for personalized dosing of INH using sampling at 2 and $4 \mathrm{~h}$ or 2,4 , and $6 \mathrm{~h}$ after dosing. These approaches will allow for dose adjustments of INH in programmatic TB treatment.

The structure of the final PK model is comparable to those previously described $[5,17,21]$. The estimated clearances and proportion of fast NAT2 acetylators described in this model are very similar to those previously described [17]. As in other published INH models, our model could only separate two of the three acetylator subgroups $[17,18]$. We did not differentiate between acetylator proportions for different ethnicities, which are known to vary [47]. The other model parameters are also mostly similar to those described previously [5, 17]. The estimates for the peripheral volume and intercompartmental clearance for INH vary between the different models. During the model building process, we encountered instability problems, making it difficult to estimate some of the model parameters. Explanations for this could be the large number of different data sources included in the model building dataset and the model complexity. The VPCs of the model are acceptable and it has been shown b

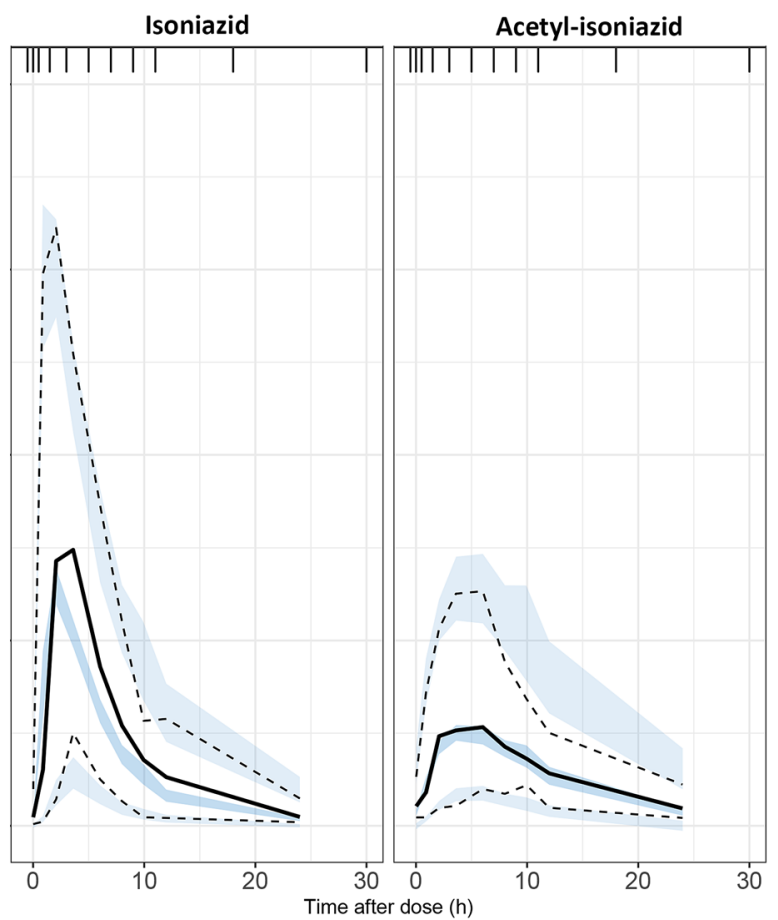

The solid lines are the $y=0$ lines, and the dashed lines define the range between which you want the majority of the observations to lie. d The normalised prediction distribution errors plotted against the time after dose. The solid lines are the $y=0$ lines and the blue lines are the means per time bin

that the model performs well for the purpose of TDM. As such, we do not see the instability as a sign of an underlying problem. We opted to have a limited inclusion of covariates in view of the purpose of this model. For other purposes, the model could probably be improved by the inclusion of additional covariates. While inter-occasion variability is known to be present for INH, we were unable to identify it in our model $[4,17]$. It has been described that inter-occasion variability may impact the predictive performance of modelbased dosing algorithms [48]. Despite this, we showed in the fit-for-purpose evaluation using multiple occasions that the model performed well.

The LSS with sampling at 2 and $4 \mathrm{~h}$ after dosing was selected as the most suitable strategy. Previously, we introduced a model-based TDM approach for rifampicin, just like INH a pillar within the TB treatment [28]. This approach also uses a LSS with sampling at 2 and $4 \mathrm{~h}$ after dosing, which means that the method described here and for rifampicin are compatible in clinical practice. In this study, we decided to develop a new PK model rather than evaluate existing models like we did for the model-based approach for rifampicin. This decision was based on the added value of a model built on a large and diverse dataset. A better LSS could potentially have been found using an optimal design 

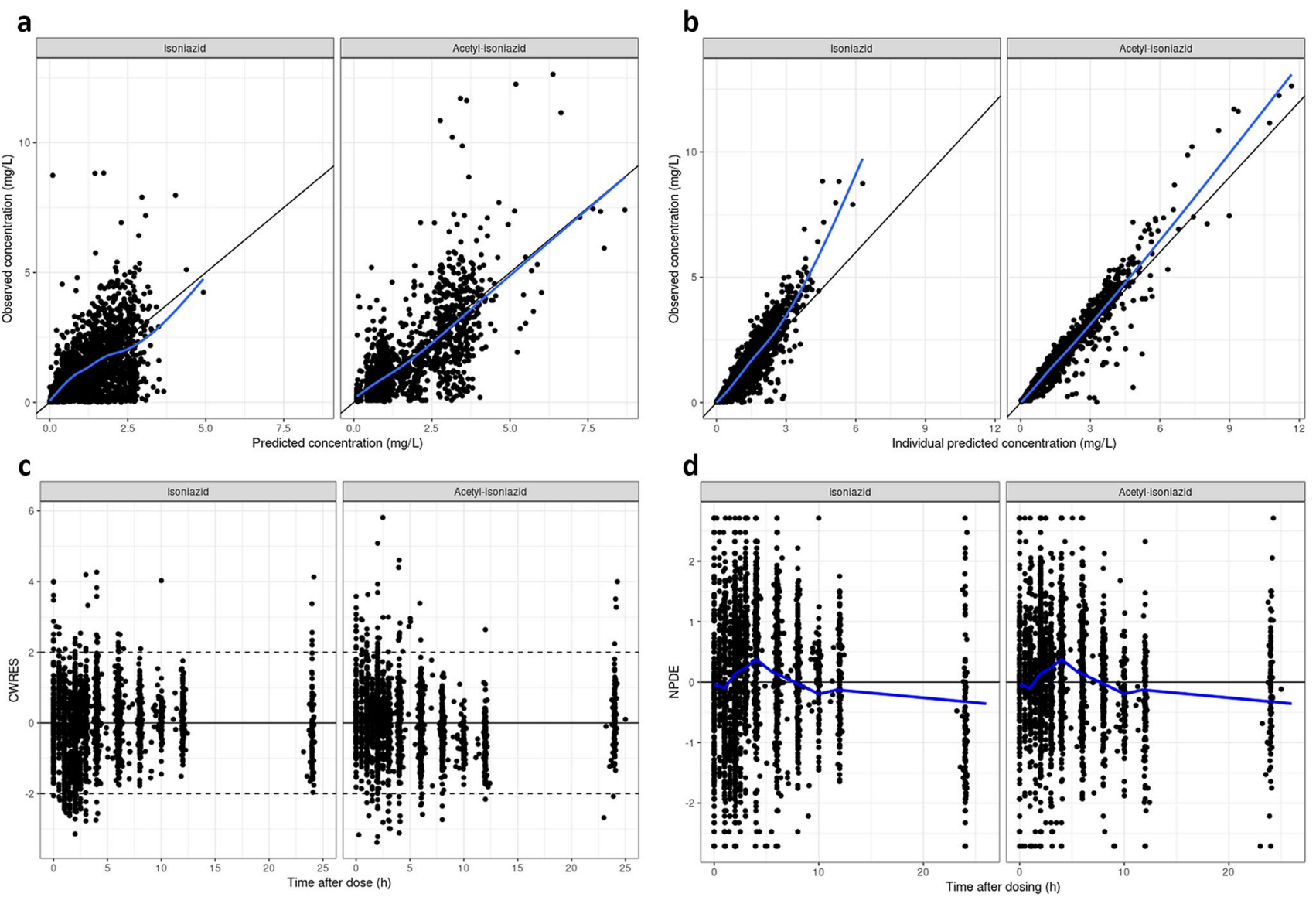

Fig. 3 Visual predictive checks of the final isoniazid population pharmacokinetic model for a slow metabolizers and $\mathbf{b}$ fast metabolizers. The visual predictive checks are based on 1000 simulations and prediction corrected. The PsN mixture option was used and the plots are based on the individual probability for belonging to a subpopulation

[36]. The solid lines represent the mean of the observed concentrations and the dashed lines represent the 2.5th and 97.5th percentiles. The shaded areas represent the $95 \%$ confidence interval of the 2.5 th, 50th, and 97.5th percentiles of the simulated concentrations

Table 3 Predictive performance comparison of the limited sampling strategies

\begin{tabular}{|c|c|c|c|c|c|}
\hline \multirow[t]{2}{*}{ Data used for prediction } & \multirow[t]{2}{*}{ Sampling strategy } & \multicolumn{2}{|l|}{$\mathrm{AUC}_{0-24}$} & \multicolumn{2}{|l|}{$C_{\max }$} \\
\hline & & RMSE, \% (SD) & ME, \% (95\% CI) & RMSE, \% (SD) & ME, \% (95\% CI) \\
\hline No data & No sampling & $73.7(4.0)$ & $55.8(48.5,63.1)$ & $44.1(2.4)$ & $25.3(19.9,30.8)$ \\
\hline \multirow[t]{3}{*}{$\mathrm{INH}+\mathrm{AcINH}$} & 2,4 , and $6 \mathrm{~h}$ & $21.8(1.2)$ & $1.6(-1.7,4.8)$ & $24.2(1.3)$ & $4.2(0.6,7.8)$ \\
\hline & 2 and $4 \mathrm{~h}$ & $23.4(1.3)$ & $-1.1(-4.6,2.4)$ & $23.8(1.3)$ & $2.7(-0.9,6.3)$ \\
\hline & $2 \mathrm{~h}$ & $34.0(1.8)$ & $10.0(5.1,14.9)$ & $26.5(1.4)$ & $6.0(2.1,9.9)$ \\
\hline \multirow[t]{3}{*}{ INH } & 2,4 , and $6 \mathrm{~h}$ & $20.9(1.1)$ & $1.7(-1.5,4.8)$ & $23.8(1.3)$ & $1.2(-2.4,4.8)$ \\
\hline & 2 and $4 \mathrm{~h}$ & $28.9(1.6)$ & $-1.8(-6.2,2.6)$ & $23.5(1.3)$ & $0.1(-3.5,3.7)$ \\
\hline & $2 \mathrm{~h}$ & $59.1(3.2)$ & $36.1(29.1,43.2)$ & $27.0(1.5)$ & $11.7(8.0,15.4)$ \\
\hline
\end{tabular}

Both the ME and RMSE are depicted as a percentage of the mean of the corresponding model-estimated parameter using the full pharmacokinetic curve

$A c I N H$ acetyl-isoniazid, $A U C_{0-24}$ area under the concentration-time curve from 0 to $24 \mathrm{~h}, C I$ confidence interval, $C_{\text {max }}$ peak concentration, $I N H$ isoniazid, $M E$ mean error, $R M S E$ root mean square error, $S D$ standard deviation 
experiment. However, by sticking to predefined sampling times, we were able to evaluate the proposed strategy using the existing time points in the dataset.

For unbiased predictions using the 2- and 4-h LSS, acetyl-INH concentration data were needed. If using this LSS without acetyl-INH PK data input, the performance of predicting the $\mathrm{AUC}_{24}$ will be lower. However, if acetyl-INH data are not available, it can be compensated for by adding a 6-h sampling time. Removing the mixture component and re-estimating the model parameters shows that not accounting for the polymorphic clearance of INH does not have a major impact on performance of the exposure prediction. After removing the mixture component, the variability caused by the polymorphic NAT2 clearance is described by an IIV more than two times the size before removing the mixture component. This increase in variability probably compensates for the lack of mixture model and prevents a significant drop in performance of the LSS.

While we present a sampling strategy using 2- and 4-h sampling times, deviation from these sampling times does not mean exposure prediction is not trustworthy anymore. The presented strategy should be seen as a flexible sampling strategy. Using sampling times that deviate from the LSS is one of the benefits of using a model-based approach. However, we did not evaluate the impact of deviating from the proposed sampling times as such sampling time deviations were not sufficiently present in the data because of the regulated nature of the included studies. It would have been possible to simulate deviating sampling times as input for the sampling strategies, but this was beyond the purpose of this study.

We tested if the LSS was fit for purpose by assessing the performance of predicting the exposure of a future sampling occasion. We showed that the strategy is able to explain most of the variability in the pharmacokinetics by comparing its performance to that without sampling. The explained variability is determined by IIV as inter-occasion variability was not included in the model. By incorporating inter-occasion variability in the model, the performance of predicting a future occasion could be further improved [48]. Prospective evaluation of this method is needed to show how well it will perform in a real TDM setting.

The model-based TDM approach has advantages, but is also a complex methodology in terms of software usage and underlying theory [49]. For this reason, implementation of a model-based TDM approach in clinical practice should be combined with a user-friendly interface to improve the ease of use. Furthermore, the translation from the modelbased results to clinical advice is crucial for successful implementation.

\section{Conclusions}

We developed a model-based LSS using INH and acetylINH data from sampling at 2 and $4 \mathrm{~h}$ after dosing to be used for individualized dosing in TDM practice. Alternatively, a 2-, 4-, and 6-h LSS can be used if only collecting PK data on INH and not on acetyl-INH. Prospective evaluation of this strategy will show how it performs in a clinical TDM setting.

Supplementary Information The online version contains supplementary material available at https://doi.org/10.1007/s40262-020-00971-2.

\section{Declarations}

Funding No funding was received specifically for this study or for the preparation of this article. Elin M. Svensson is supported by PanACEA, which is part of the European and Developing Countries Clinical Trials Partnership (EDCTP) 2 programme supported by the European Union (Grant number TRIA2015-1102-PanACEA).

Conflict of interest All authors declare no conflicts of interest that could have influenced the submitted work.

Ethical approval The data used in this study was collected in line with the principles of the Declaration of Helsinki. Approval was granted by institutional review boards and independent ethics committees for each study of which data was used in this work.

Consent to participate Not applicable.

Consent for publication Not applicable.

Availability of data and material Not applicable.

Code availability The model code is available within the supplemental materials.

Author contributions $\mathrm{SvB}$, RtH and ES performed the analysis, data interpretation and writing of the manuscript. JA, CME and RE were involved in data collection, data interpretation and writing of the manuscript. MB, EB, RD, AD, SG, CM, NN, NH, WH, MH, GK, KR, IS, HS and AT were involved in data collection and contributed to the writing of the manuscript.

Open Access This article is licensed under a Creative Commons Attribution-NonCommercial 4.0 International License, which permits any non-commercial use, sharing, adaptation, distribution and reproduction in any medium or format, as long as you give appropriate credit to the original author(s) and the source, provide a link to the Creative Commons licence, and indicate if changes were made. The images or other third party material in this article are included in the article's Creative Commons licence, unless indicated otherwise in a credit line to the material. If material is not included in the article's Creative Commons licence and your intended use is not permitted by statutory regulation or exceeds the permitted use, you will need to obtain permission directly from the copyright holder. To view a copy of this licence, visit http://creativecommons.org/licenses/by-nc/4.0/. 


\section{References}

1. World Health Organization. Global tuberculosis report 2019. http://www.who.int/tb/publications/global_report/en/. Accessed 7 Dec 2020.

2. Alsultan A, Peloquin CA. Therapeutic drug monitoring in the treatment of tuberculosis: an update. Drugs. 2014;74(8):839-54.

3. World Health Organization. Essential medicines list. 20th ed. 2017. http://www.who.int/medicines/publications/essentialmedici nes/en/. Accessed 7 Dec 2020.

4. Wilkins JJ, Landon G, McIlleron H, et al. Variability in the population pharmacokinetics of isoniazid in South African tuberculosis patients. Br J Clin Pharmacol. 2011;72(1):51-62.

5. Sundell J, Bienvenu E, Janzén D, et al. Model-based assessment of variability in isoniazid pharmacokinetics and metabolism in patients co-infected with tuberculosis and HIV: implications for a novel dosing strategy. Clin Pharmacol Ther. 2020;108(1):73-80.

6. Pasipanodya JG, Srivastava S, Gumbo T. Meta-analysis of clinical studies supports the pharmacokinetic variability hypothesis for acquired drug resistance and failure of antituberculosis therapy. Clin Infect Dis. 2012;5(2):169-77.

7. Sturkenboom MG, Akkerman OW, van Altena R, et al. Dosage of isoniazid and rifampicin poorly predicts drug exposure in tuberculosis patients. Eur Respir J. 2016;48(4):1237-9.

8. Parkin DP, Vandenplas S, Botha FJ, et al. Trimodality of isoniazid elimination: phenotype and genotype in patients with tuberculosis. Am J Respir Crit Care Med. 1997;155(5):1717-22.

9. Jayaram R, Shandil RK, Gaonkar S, et al. Isoniazid pharmacokinetics-pharmacodynamics in an aerosol infection model of tuberculosis. Antimicrob Agents Chemother. 2004;48(8):2951-7.

10. Jayaram R, Gaonkar S, Kaur P, et al. Pharmacokinetics-pharmacodynamics of rifampin in an aerosol infection model of tuberculosis. Antimicrob Agents Chemother. 2003;47(7):2118-24.

11. Gumbo T, Louie A, Liu W, et al. Isoniazid bactericidal activity and resistance emergence: integrating pharmacodynamics and pharmacogenomics to predict efficacy in different ethnic populations. Antimicrob Agents Chemother. 2007;51(7):2329-36.

12. Pasipanodya JG, McIlleron H, Burger A, et al. Serum drug concentrations predictive of pulmonary tuberculosis outcomes. J Infect Dis. 2013;208(9):1464-73.

13. Rockwood N, Pasipanodya JG, Denti P, et al. Concentrationdependent antagonism and culture conversion in pulmonary tuberculosis. Clin Infect Dis. 2017;64(10):1350-9.

14. Magis-Escurra C, Later-Nijland HMJ, Alffenaar JWC, et al. Population pharmacokinetics and limited sampling strategy for first-line tuberculosis drugs and moxifloxacin. Int J Antimicrob Agents. 2014;44(3):229-34.

15. Maze MJ, Paynter J, Chiu W, et al. Therapeutic drug monitoring of isoniazid and rifampicin during anti-tuberculosis treatment in Auckland, New Zealand. Int J Tuberc Lung Dis. 2016;20(7):955-60.

16. Zuur MA, Pasipanodya JG, van Soolingen D, et al. Intermediate susceptibility dose-dependent breakpoints for highdose rifampin, isoniazid, and pyrazinamide treatment in multidrug-resistant tuberculosis programs. Clin Infect Dis. 2018;67(11):1743-9.

17. Chirehwa MT, McIlleron H, Wiesner L, et al. Effect of efavirenz-based antiretroviral therapy and high-dose rifampicin on the pharmacokinetics of isoniazid and acetyl-isoniazid. J Antimicrob Chemother. 2019;74(1):139-48.

18. Denti P, Jeremiah K, Chigutsa E, et al. Pharmacokinetics of isoniazid, pyrazinamide, and ethambutol in newly diagnosed pulmonary TB patients in Tanzania. PLoS ONE. 2015;10(10):e0141002.
19. Lalande L, Bourguignon L, Bihari S, et al. Population modeling and simulation study of the pharmacokinetics and antituberculosis pharmacodynamics of isoniazid in lungs. Antimicrob Agents Chemother. 2015;59(9):5181-9.

20. Peloquin CA, Jaresko GS, Yong AC, et al. Population pharmacokinetic modeling of isoniazid, rifampin, and pyrazinamide. Antimicrob Agents Chemother. 1997;41(12):2670-9.

21. Seng KY, Hee KH, Soon GH, et al. Population pharmacokinetic analysis of isoniazid, acetylisoniazid, and isonicotinic acid in healthy volunteers. Antimicrob Agents Chemother. 2015;59(11):6791-9.

22. Zvada SP, Denti P, Donald PR, et al. Population pharmacokinetics of rifampicin, pyrazinamide and isoniazid in children with tuberculosis: in silico evaluation of currently recommended doses. J Antimicrob Chemother. 2014;69(5):1339-49.

23. Semvua HH, Mtabho CM, Fillekes Q, et al. Efavirenz, tenofovir and emtricitabine combined with first-line tuberculosis treatment in tuberculosis-HIV-coinfected Tanzanian patients: a pharmacokinetic and safety study. Antivir Ther. 2013;18(1):105-13.

24. Tostmann A, Mtabho CM, Semvua HH, et al. Pharmacokinetics of first-line tuberculosis drugs in Tanzanian patients. Antimicrob Agents Chemother. 2013;57(7):3208-13.

25. Burhan E, Ruesen C, Ruslami R, et al. Isoniazid, rifampin, and pyrazinamide plasma concentrations in relation to treatment response in Indonesian pulmonary tuberculosis patients. Antimicrob Agents Chemother. 2013;57(8):3614-9.

26. Aarnoutse RE, Kibiki GS, Reither K, et al. Pharmacokinetics, tolerability, and bacteriological response of rifampin administered at 600, 900, and 1,200 milligrams daily in patients with pulmonary tuberculosis. Antimicrob Agents Chemother. 2017;61(11):e01054-17.

27. Boeree MJ, Heinrich N, Aarnoutse RE, et al. High-dose rifampicin, moxifloxacin, and SQ109 for treating tuberculosis: a multi-arm, multi-stage randomised controlled trial. Lancet Infect Dis. 2017;17(1):39-49.

28. van Beek SW, ter Heine R, Keizer RJ, et al. Personalized tuberculosis treatment through model-informed dosing of rifampicin. Clin Pharmacokinet. 2019;58(6):815-26.

29. Sturkenboom MG, van der Lijke H, Jongedijk EM, et al. Quantification of isoniazid, pyrazinamide and ethambutol in serum using liquid chromatography-tandem. J Appl Bioanal. 2015;1(3):89-98.

30. Aarnoutse RE, Sturkenboom MGG, Robijns K, et al. An interlaboratory quality control programme for the measurement of tuberculosis drugs. Eur Respir J. 2015;46(1):268-71.

31. R-Core-Team. R: a language and environment for statistical computing. 2016. https://www.R-project.org/. Accessed 7 Dec 2020.

32. Beal S, Sheiner LB, Boeckmann A, Bauer RJ. NONMEM user's guides (1989-2009). Ellicott City: Icon Development Solutions; 2009.

33. Keizer RJ, Karlsson MO, Hooker A. Modeling and simulation workbench for NONMEM: tutorial on Pirana, PsN, and Xpose. CPT Pharmacometrics Syst Pharmacol. 2013;2(6):e50.

34. Svensson E, van der Walt JS, Barnes KI, et al. Integration of data from multiple sources for simultaneous modelling analysis: experience from nevirapine population pharmacokinetics. Br J Clin Pharmacol. 2012;74(3):465-76.

35. Anderson BJ, Holford NH. Mechanism-based concepts of size and maturity in pharmacokinetics. Annu Rev Pharmacol Toxicol. 2008;48:303-32.

36. Carlsson KC, Savić RM, Hooker AC, et al. Modeling subpopulations with the \$MIXTURE subroutine in NONMEM: finding the individual probability of belonging to a subpopulation for the use in model analysis and improved decision making. AAPS J. 2009;11(1):148-54. 
37. Beal SL. Ways to fit a PK model with some data below the quantification limit. J Pharmacokinet Pharmacodyn. 2001;28(5):481-504.

38. Dosne AG, Bergstrand M, Harling K, et al. Improving the estimation of parameter uncertainty distributions in nonlinear mixed effects models using sampling importance resampling. $\mathbf{J}$ Pharmacokinet Pharmacodyn. 2016;43(6):583-96.

39. Dosne AG, Bergstrand M, Karlsson MO. An automated sampling importance resampling procedure for estimating parameter uncertainty. J Pharmacokinet Pharmacodyn. 2017;44(6):509-20.

40. Bergstrand M, Hooker AC, Wallin JE, et al. Prediction-corrected visual predictive checks for diagnosing nonlinear mixed-effects models. AAPS J. 2011;13(2):143-51.

41. Arshad U, Chasseloup E, Nordgren R, et al. Development of visual predictive checks accounting for multimodal parameter distributions in mixture models. J Pharmacokinet Pharmacodyn. 2019;46(3):241-50.

42. Magis-Escurra C, van den Boogaard J, Ijdema D, et al. Therapeutic drug monitoring in the treatment of tuberculosis patients. Pulmon Pharmacol Ther. 2012;25(1):83-6.

43. Sheiner LB, Beal SL. Some suggestions for measuring predictive performance. J Pharmacokinet Biopharm. 1981;9(4):503-12.

44. Faber NM. Estimating the uncertainty in estimates of root mean square error of prediction: application to determining the size of an adequate test set in multivariate calibration. Chemometric Intell Lab Syst. 1999;49(1):79-89.

45. Keizer RJ, ter Heine R, Frymoyer A, et al. Model-informed precision dosing at the bedside: scientific challenges and opportunities. CPT Pharmacometrics Syst Pharmacol. 2018;7(12):785-7.

46. Gordi T, Xie R, Huong NV, et al. A semiphysiological pharmacokinetic model for artemisinin in healthy subjects incorporating autoinduction of metabolism and saturable first-pass hepatic extraction. Br J Clin Pharmacol. 2005;59(2):189-98.

47. Aarnoutse R. Pharmacogenetics of antituberculosis drugs Antituberculosis Chemother. 2011;40:176-90.

48. Abrantes JA, Jönsson S, Karlsson MO, et al. Handling interoccasion variability in model-based dose individualization using therapeutic drug monitoring data. Br J Clin Pharmacol. 2019;85(6): 1326-36.

49. Donagher J, Martin JH, Barras MA. Individualised medicine: why we need Bayesian dosing. Intern Med J. 2017;47(5):593-600. 